\title{
Prevalencia y factores de riesgo de hipertensión arterial en escolares mexicanos: caso Sabinas Hidalgo
}

\author{
Enrique Oliver Aregullin-Eligio, Ped, (I) María Cándida Alcorta-Garza, Ped.(I)
}

\author{
Aregullin-Eligio EO, Alcorta-Garza MC. \\ Prevalencia y factores de riesgo de hipertensión arterial \\ en escolares mexicanos: caso Sabinas Hidalgo. \\ Salud Publica Mex 2009;5I:I4-18.
}

\begin{abstract}
Resumen
Objetivo. Determinar la prevalencia de hipertensión arterial (HTA) y los factores de riesgo asociados en escolares de 6 a 12 años, con la metodología aceptada. Material y métodos. Se examinaron 329 alumnos de educación primaria para investigar asociaciones entre HTA, índice de masa corporal (IMC), edad y género, usando un estudio transversal. Se midió la presión arterial y se utilizó el percentil 95 para diagnosticar HTA. Se compararon niños con y sin HTA. Se utilizaron las razones de momios simple y ajustada con la $X^{2}$ de MantelHaenszel como medidas de asociación. Resultados. Los niños con sobrepeso (IMC $\geq 85$ percentil) y del grupo de I I - I 2 años tuvieron al menos cuatro veces más posibilidades de HTA después de controlar variables confusoras. Conclusiones. Los niños que presentaron HTA ascendieron a $4.9 \%$, con mayor prevalencia entre aquellos con sobrepeso y pertenecientes a un grupo etario mayor. Las asociaciones encontradas pueden ser marcadores de HTA en niños mexicanos.
\end{abstract}

Palabras clave: hipertensión; prevalencia; niños; México
Aregullin-Eligio EO,Alcorta-Garza MC.

Prevalence and risk factors of high blood pressure in Mexican school children in Sabinas Hidalgo.

Salud Publica Mex 2009;5 I: I4-I8.

\section{Abstract}

Objective. To determine the prevalence and associated risk factors of high blood pressure (HBP) in school children 6 to 12 years of age with the accepted methodology. Material and Methods. Elementary school children were examined $(n=329)$. Associations between HBP, body mass index (BMI), age and gender were investigated using a cross-sectional study. Blood pressure was measured and the 95th percentile was used to determine HBP. Comparisons between children with and without HBP were made. The crude and $\mathrm{X}^{2}$ Mantel-Haenszel adjusted odds ratios were used as measures of association. Results. Overweight children (BMI $\geq 85$ th percentile) and those in the age group I I- 12 years were at least four times as likely to present with HBP after controlling for confounding factors. Conclusion. A total of $4.9 \%$ of school children had HBP, with a higher prevalence occurring among overweight and the older group.The associations found here can be used as markers for an increased likelihood of HBP in Mexican children.

Key words: hypertension; children; prevalence; Mexico

(I) Departamento de Pediatría, Hospital General de Sabinas Hidalgo Virginia Ayala de Garza. Servicios de Salud de Nuevo León, Sabinas Hidalgo. Nuevo León, México.

Fecha de recibido: 7 de enero de 2008 • Fecha de aprobado: 30 de septiembre de 2008 Solicitud de sobretiros: Dr. Enrique Oliver Aregullin Eligio. Prof. Alberto Chapa 550, col. Bellavista. 65270 Sabinas Hidalgo, Nuevo León, México. Correo electrónico: oliveraregullin@hotmail.com 
$\mathrm{O}_{\mathrm{h}}^{\mathrm{th}}$ trora considerada como un padecimiento raro, la hipertensión arterial (HTA) en niños se ha vuelto cada vez más común y ha sido reconocida ampliamente como un problema de salud pública; ${ }^{1}$ la prevalencia notificada por varios autores va de 5.4 a $21 \% .^{2-5}$ En los últimos años ha aumentado el interés en la detección de HTA en niños y al respecto se han llevado a cabo estudios formales de grandes cohortes; ${ }^{5-7}$ incluso, se ha profundizado en el entendimiento de los mecanismos moleculares y de la patogénesis de los trastornos genéticos que causan HTA en el niño. ${ }^{8}$ La HTA pediátrica puede ser secundaria o esencial. Las nefropatías, la coartación de aorta y las enfermedades endocrinas son causas comunes de HTA en niños. Sin embargo, como en los adultos, la mayoría de los niños tienen HTA primaria. Además, se ha demostrado que la HTA en niños correlaciona con historia familiar y sobrepeso. De gran relevancia resulta el hecho de que muchas de las complicaciones (hipertrofia ventricular izquierda, aterosclerosis) pudieran originarse incipientemente en la infancia ${ }^{9,10}$ y que las cifras tensionales infantiles se correlacionan con las del adulto.,11 La edad, sexo, talla, raza, estrato socioeconómico y obesidad son factores previamente reconocidos que afectan la presión arterial (PA). ${ }^{5,9}$ Por lo tanto, aun teniendo una prevalencia menor que en la edad adulta, la detección de HTA en niños tiene una gran importancia en la evaluación clínica y está recomendada de forma rutinaria (control niño sano) en niños desde los tres años de edad. ${ }^{12}$ En diversos estudios realizados en México, se notifican prevalencias entre 1 y $10 \%$; sin embargo, la metodología de medición no ha sido consistente entre los mismos. ${ }^{13-15} \mathrm{El}$ grupo de trabajo de hipertensión en niños y adolescentes del Programa Nacional de Educación en Hipertensión Arterial y la Academia Americana de Pediatría (AAP) ha emitido nuevas recomendaciones sobre la metodología de medición y las tablas de valores normales, ${ }^{12}$ mismas que han sido adoptadas por estudios recientes. ${ }^{2,16} \mathrm{En}$ este trabajo se adoptaron esas recomendaciones, por vez primera en el medio, para determinar la prevalencia de HTA en niños mexicanos, y se examinaron las potenciales asociaciones entre factores como índice de masa corporal (IMC), sexo y grupo de edad. El objetivo era determinar la prevalencia de HTA y examinar los factores de riesgo asociados en escolares de 6 a 12 años de edad en la ciudad de Sabinas Hidalgo, Nuevo León, usando la metodología propuesta por la AAP.

\section{Material y métodos}

Los datos se obtuvieron a partir de mediciones en niños de las escuelas primarias "Anexa a la normal" y "Manuel M. García" de Sabinas Hidalgo, comunidad del norte del estado de Nuevo León, muy cercana a la frontera entre México y los Estados Unidos de América, entre febrero y junio de 2007. Se utilizó un procedimiento de muestreo no probabilístico para reflejar los aspectos socioeconómicos de la zona escolar, de cuyo número total ( $n=3$ 996) se obtuvo una muestra de 330 niños entre los 6 y los 12 años de edad. Únicamente dos niños (de 13 y 15 años) salieron de ese rango y fueron agregados al grupo de edad más cercano. Para el cálculo del tamaño de la muestra ${ }^{16}$ se empleó un nivel de significancia de $99 \%$, con una precisión de $5 \%$ y una prevalencia calculada de $10 \%$, obteniendo la cifra de 237. No obstante, para este estudio se reclutaron 330 niños, de los cuales se eliminó a uno por no presentar datos completos en la hoja de encuesta, de tal manera que la cifra definitiva fue de 329 niños. El protocolo de investigación fue evaluado y aprobado por el comité de ética del Hospital General de Sabinas Hidalgo. Se proporcionó a las autoridades educativas una descripción detallada de la importancia del estudio y de los procedimientos a realizarse y se obtuvo la autorización correspondiente. Además, al inicio del estudio se obtuvo el consentimiento informado de cada niño. La hoja de encuesta, que fue llenada por asistentes del investigador, incluyó datos acerca del nombre, edad, género, peso, talla, IMC, perímetro braquial, tensión arterial sistólica y diastólica (TAS y TAD respectivamente) y observaciones. Se consignó, además, si el niño estaba bajo algún tratamiento médico, o si se sabía portador de HTA. Los datos se obtuvieron del examen físico y de los rubros del cuestionario.

La clasificación de los percentiles de PA para este estudio se determinó usando tablas normativas para la edad, género, y estatura de cada niño, generadas por el grupo de trabajo de hipertensión en niños y adolescentes del Programa Nacional de Educación de Hipertensión y la AAP. ${ }^{12}$ El percentil 95 se empleó para diagnosticar HTA para la edad, género y estatura de cada niño.

Se pesó a los niños vistiendo sus uniformes escolares y estando descalzos. Se usó una báscula Nuevo León tipo clínica, con estadímetro, calibrada. La talla se midió en el estadímetro de la misma báscula, con el niño de pie, en el centro de la plataforma y con los brazos a ambos lados del cuerpo. Todas las mediciones se realizaron entre las 9:00 y las 12:00 horas. El peso se midió en kilos y la talla en metros, para calcular el IMC, el cual se determina dividiendo el peso $(\mathrm{kg})$ entre la talla al cuadrado $\left(\mathrm{m}^{2}\right)$. Los niños con un IMC $\geq 85$ percentil para edad y sexo fueron clasificados con sobrepeso. ${ }^{17}$ Para propósitos de este estudio las categorías sobrepeso y obesidad se combinaron bajo el nombre de sobrepeso.

La PA se midió después de, al menos, cinco minutos de reposo, estando el niño sentado en un asiento con 
soporte para la espalda; la medición se tomó en el brazo derecho y en un ambiente tranquilo, dentro de las instalaciones de las escuelas primarias. Se usaron esfingomanómetros calibrados aneroides (Welch Allyn, Adex) con manguitos de las dimensiones estándar recomendadas ${ }^{12}$ (el ancho de la bolsa del manguito debe ser por lo menos $40 \%$ de la circunferencia del brazo en un punto medio entre el olécranon y el acromion y la longitud debe cubrir 80 a 100\% de la circunferencia del mismo), con una índice ancho: longitud de al menos 1:2. Para este propósito se determinaron las circunferencias braquiales $(\mathrm{CB})$ antes de la toma de PA, y con base en éstas se decidió el manguito correspondiente: manguito de $9 \times 21 \mathrm{~cm}$. (CB hasta $22 \mathrm{~cm}$.); de $10 \times 24 \mathrm{~cm}$. (CB hasta $26 \mathrm{~cm}$ ), y de $13 \times 31 \mathrm{~cm}$. (CB hasta $34 \mathrm{~cm}$.). La tensión arterial sistólica (TAS) se determinó por la auscultación del primer ruido de Korotkoff (k1) y la tensión arterial diastólica (TAD) por la del quinto ruido (k5). ${ }^{12,18}$ De acuerdo con el criterio establecido por el informe de diagnóstico, evaluación y tratamiento de HTA en niños y adolescentes, en este estudio la HTA en niños fue definida como el promedio de TAS y/o TAD $\geq 95$ percentil para edad, género y talla, basados en tablas normativas, medidas en, al menos, tres ocasiones diferentes. La prehipertensión en niños se definió como un promedio de TAS o TAD $\geq 90$ percentil pero $<95$ percentil. Por lo tanto, el punto de corte para determinar HTA en este estudio fue el percentil $95 .^{12}$

En los niños que presentaron lecturas elevadas o en rango de hipertensión (percentiles $\geq 90$ y $\geq 95$, respectivamente) se hizo una segunda lectura después de 10 minutos adicionales de reposo. Los niños cuyos valores permanecieron aún en el rango de hipertensión (percentil 295 ), fueron sometidos a dos mediciones adicionales, cuatro meses después, en días diferentes y bajo las mismas circunstancias.

\section{Metodología estadística}

El análisis de los datos se llevó a cabo con el paquete SPSS (SPSS 14.0 para Windows). Se definió la prevalencia de HTA como la prevalencia de TAS y TAD $\geq 95$ percentil, o la prevalencia aislada de TAS o TAD $\geq 95$ percentil, y los datos fueron computados para compararlos con niños con PA $<95$ percentil en busca de asociación de posibles variables. Se utilizó la razón de momios $(R M)$ como medida de asociación. Se obtuvieron $R M$ de $P A \geq 95$ percentil con variables como sobrepeso, género y grupo de edad. Se uso el procedimiento de Mantel y Haenszel ${ }^{16,19}$ para evaluar la asociación de cada variable y $P A \geq 95$ percentil, ajustando las $R M$ y controlando simultáneamente las potenciales variables de refutación ( $R M$ ajustada). Se calcularon intervalos de confianza (IC) de $95 \%$ para estas asociaciones. Se obtuvo la relación bivariada entre la TA sistólica y diastólica y el IMC ajustándose para la edad y se usó la prueba de Kruskal-Wallis para obtener la significancia estadística de estas relaciones.

\section{Resultados}

El rango de edad de los niños participantes fue de 6-12 años, y la prevalencia de sobrepeso de $39.2 \%$. Ningún sujeto estaba tomando medicamento ni se sabía portador de HTA al momento de las mediciones. Para propósitos de este estudio, todos los niños con $\mathrm{TA} \geq 95$ percentil, ya sea TAS aislada o acompañada por TAD $\geq 95$ percentil, fueron agrupados en la categoría de HTA, cuya prevalencia general fue de $4.9 \%$ tal cual se muestra en el cuadro I; asimismo se muestran la $R M$ y la $R M$ ajustada para las asociaciones entre HTA y las variables de interés. En el grupo de edad de 11-12 años la RM mostró una probabilidad seis veces mayor de tener HTA; no obstante, esa probabilidad disminuyó a cuatro veces después del ajuste con las potenciales variables de confusión ( $R M$ ajustada $=4.88$; IC 1.4-16.6, $p=0.005$ ). La probabilidad de ser hipertenso fue siete veces más alta en los niños con sobrepeso ( $R M$ ajustada $=7.43$; IC 1.75-31.5, $p<0.001$ ). En la relación bivariada de los valores de presión arterial sistólica y diastólica con el IMC ajustado por edad, se observó que para el grupo de edad de niños con obesidad, la presión sistólica es mayor y estadísticamente diferente (figura 1) a la correspondiente a los niños con sobrepeso o peso normal $(p=0.006)$. Estas diferencias no se observaron para la presión sistólica $(p=0.128)$.

\section{Discusión}

Trabajos previos han mostrado una prevalencia de HTA infantil que va de 1.2 a 13\% y, especialmente en México, de 1 a $10 \% \cdot{ }^{13-15}$ La muestra del presente estudio revela una prevalencia de $4.9 \%$ en niños escolares. Estos resultados sugieren, como en otros estudios, que la HTA en niños no es rara. La prevalencia de sobrepeso/obesidad fue de $39.2 \%$, esto es, más alta que la notificada en niños escolares de todo el país. ${ }^{20}$ En contraste con estudios previos, la metodología usada en este estudio es consistente con las actuales recomendaciones del grupo de trabajo de hipertensión en niños y adolescentes del Programa Nacional de Educación en Hipertensión y la $\mathrm{AAP}_{r}^{12}$ cuyas gráficas normativas incluyen datos de niños hispanos. A diferencia de otros trabajos ${ }^{13-15}$ la importancia del uso del tamaño adecuado de los manguitos ha sido destacada. Autores nacionales y extranjeros han reconocido esta metodología como la más adecuada ${ }^{2,18,21}$ y al usarla se buscó estandarizar la muestra con las correspondientes a los estudios más 
Cuadro I

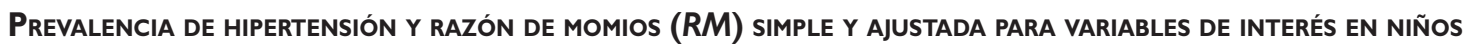
escolares. Sabinas Hidalgo, Nuevo León, México. Enero 2008

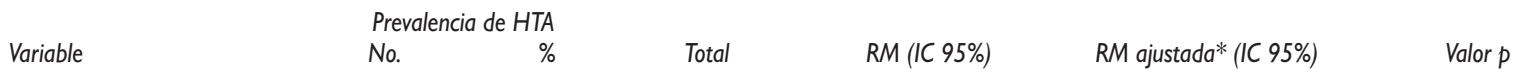

Grupo de edad (años)

\begin{tabular}{lrrrrrr}
6 a 8 & 3 & 2.0 & 147 & 1 & 1 & \\
\hline 9 a 10 & 3 & 3.2 & 95 & $1.56(0.3-7.9)$ & $0.49(0.1-1.5)$ & $p=0.681$ \\
\hline II a 12 & 10 & 11.5 & 87 & $6.23(1.6-23.3)$ & $4.88(1.4-16.6)$ & $p=0.005$ \\
\hline Total & 16 & 4.9 & 329 & & &
\end{tabular}

Género

$\begin{array}{ccccccc}\text { Masculino } & 5 & 3.4 & 146 & 1 & 1 & 1.84(0.54-6.28)\end{array}$

IMC

\begin{tabular}{lrrrrrr} 
Sobrepeso/obesidad & 13 & 10.1 & 129 & $7.35(2-26.3)$ & $7.43(1.75-31.5)$ & I \\
\hline Normal & 3 & 1.5 & 200 & 1 & 1
\end{tabular}

*RM ajustada para edad, género e IMC.

Nota: Para evaluar la multicolinealidad, cada covariable fue relacionada con las otras covariables en el modelo con un factor de inflación de varianza (VIF) de 5.27

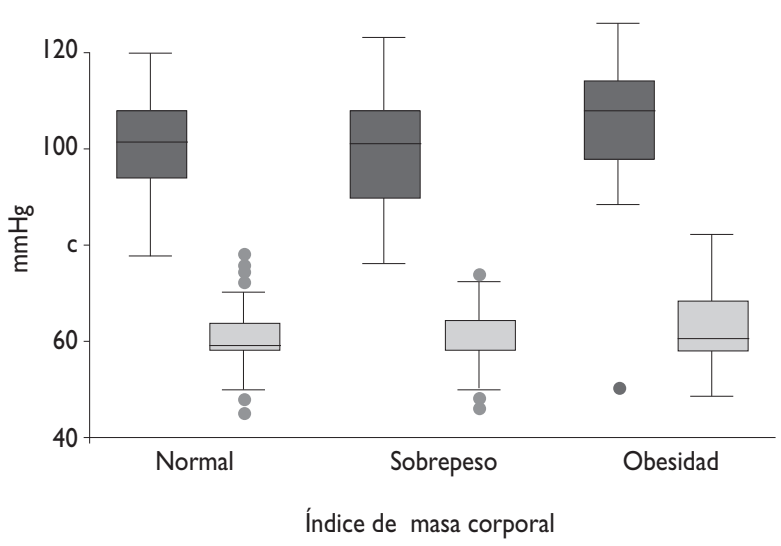

Presión arterial sistólica

Prueba de Kruskal-Wallis $p=0.006$

$\square$ Presión arterial diastólica

Prueba de Kruskal-Wallis $p=0.128$

Figura I. Distribución de LOS VALORES dE LA PRESIÓN ARTERIAL DE ACUERDO CON SU CLASIFICACIÓN ANTROPOMÉTRICA DE ÍNDICE DE MASA CORPORAL, EN UNA MUESTRA DE NIÑOS DE 6 a 12 años de EDAd del Estado de Nuevo León recientes en este campo. Al igual que en el presente estudio, algunos autores han informado mayor prevalencia de HTA en los grupos etarios mayores y aunque la prevalencia de obesidad también es mayor en este grupo etario, el ajuste de ambas variables confirma el hallazgo. En el presente trabajo el sobrepeso/ obesidad fue el factor más fuertemente ligado a HTA; este ha-

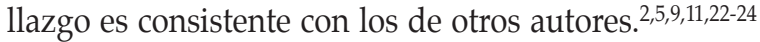
Trabajos recientes muestran que la HTA está presente en niños con sobrepeso desde etapas tempranas y que este es un factor de riesgo para enfermedad coronaria. ${ }^{25}$ En el presente estudio, el hecho de tener sobrepeso u obesidad incrementó siete veces la posibilidad de hipertensión después de ajustarse para género y grupo de edad, lo cual confirma que la obesidad es un factor fuertemente ligado a HTA en niños. Existen varios factores que pueden haber influenciado los resultados de este estudio y que son potenciales limitantes del mismo: la prevalencia de sobrepeso/obesidad en la población de estudio es más alta (39.2 vs. 26\%) que la media nacional; debido a que se trata de un estudio transversal las relaciones causa-efecto fueron evaluadas en un mismo punto en el tiempo; no se recabaron antecedentes heredofamiliares ni personales que pudieran influir en la presencia de 
HTA, como sedentarismo y grado de actividad física. La ausencia de información retrospectiva del peso de cada sujeto es un potencial factor de causalidad reversa. Sin embargo, los resultados en este estudio son consistentes con los hallazgos de otros autores en cuanto a la relación de sobrepeso y HTA y ofrecen datos relevantes para niños mexicanos con la metodología actualmente recomendada. Estos hallazgos son importantes marcadores que pueden aplicarse a grupos etarios de características similares. El 4.9\% de los niños escolares de la muestra de dos escuelas primarias del norte de Nuevo León tuvieron HTA, y resultó especialmente alta la prevalencia entre los niños con sobrepeso y los del grupo de mayor edad (11-12 años). La alta prevalencia de HTA en niños con estos marcadores está confirmada por estudios previos, así como la morbilidad en el corto plazo y las implicaciones de enfermedad cardiovascular en el largo plazo. ${ }^{3,25,26}$ Por lo tanto, un monitoreo temprano y un diagnóstico oportuno de HTA representan los pilares básicos de la prevención de este padecimiento, y donde los pediatras y los médicos que atienden niños están llamados a ser piedra angular.

\section{Agradecimientos:}

Los autores agradecen al doctor Eduardo César Lazcano Ponce, del Instituto Nacional de Salud Pública, su asesoría científica y ayuda técnica; al doctor Rosendo Chapa Contreras, director del Hospital General de Sabinas Hidalgo, las facilidades otorgadas para realizar el proyecto, y al profesor Manlio Sigifredo Perales Rodríguez, jefe de zona escolar en Sabinas Hidalgo, la autorización y facilidades brindadas para llevar a cabo el trabajo de campo.

\section{Referencias}

I. Sorof J, Daniels S. Obesity hypertension in children: a problem of epidemic proportions. Hypertension 2002; 40:44I-447.

2. Urrutia-Rojas X, Egbuchunam CU, Bae S, Menchaca J, Bayona M. High blood pressure in school children: prevalence and risk factors. BMC Pediatr 2006; 6:32.

3. Lauer RM, Clarke WR. Childhood risk factors for high adult blood pressure: the Muscatine Study. Pediatrics 1984;84:633-64I.

4. Rames L, Clarke W, Connor W. Normal blood pressures and the evaluation of sustained blood pressure elevation in childhood: the Muscatine Study. Pediatrics 1978;61:245-25I.
5. Sorof JM, Lai D, Turner J, Poffenbarger T, Potman R. Overweight, ethnicity, and the prevalence of hypertension in school-aged children. Pediatrics 2004; I I3(3):475-482.

6. National Institute of Health. Cardiovascular profile of I5 000 children of school age in three communities 1971-1975. En: DHEW Publication No.78, 1472. Washington, DC: US Government Printing Office, 1978. 7. Sinaiko AR, Gómez-Martin O, Prineas RJ. Prevalence of "significant" hipertension in junior high school-age children: the Children and Adolescent Blood Pressure Program. J Pediatr 1989; I 14: 664-669. 8.Williams SS.Advances in genetic hypertension. Curr Opin Pediatr 2007;19(2): 192-198.

9.Burke V. Obesity in childhood and cardiovascular risk. Clin Exp Pharmacol Physiol 2006; 33(9):83I-837.

10. Hansen HS, Nielsen JR, Froberg K. Left ventricular hypertrophy in children from the upper five percent of the blood pressure distributionthe Odense Schoolchild Study.J Hum Hypertens 1992;6(I):4I-45.

I I. Gillman MW, Cook NR, Rosner B. Identifying children at high risk for the development of essential hypertension. J Pediatr 1993; I22:837-847. 12. National High Blood Pressure Education Program Working Group on High Blood Pressure in Children and Adolescents: the fourth report on the diagnosis, evaluation, and treatment of high blood pressure in children and adolescents. Pediatrics 2004; I I4: 555-576.

13. Delgado J,Alcoltzin C. Cifras de tensión arterial en niños y adolescentes en Colima (tesis). México: Universidad de Colima, 1994. 14. Cervantes J,Alcoltzin C, Aguayo A. Diagnóstico y prevalencia de hipertensión arterial en menores de 19 años en la ciudad de Colima. Salud Publica Mex 2000;(42)6: 529-532.

15. Cobos O, Rubio R, García de Alba JE, Parra JZ. La presión arterial en escolares de Guadalajara. Salud Publica Mex 1983; 25: 177-183.

16. Daniel W. Bioestadística: base para el análisis de las ciencias de la salud. 4a. edición. Georgia: Limusa Wiley, 2002.

17. Rosner B, Prineas R, Loggie J. Percentiles for body mass index in US children 5 to 17 years of age.J Pediatr 1998; I32:21 I-222.

18. Diaz BLA y cols. Circunstancias especiales: hipertensión arterial en niños, hipertensión arterial en el adulto mayor, hipertensión en el embarazo. Rev Mex Cardiol 2005; 16 (I): 35-4I.

19. Bland JM,Altman DG. Statistics notes: the odds ratio. BMJ 2000; 320: 1468.

20. Olaiz-Fernández G, Rivera-Dommarco J, Shamah-Levy T, Rojas R, Villalpando-Hernández S, Hernández-Avila M, et al. Encuesta nacional de salud y nutrición 2006. Cuernavaca, México: Instituto Nacional de Salud Pública, 2006.

2I. Podoll A, Grenier M, Croix B, Feig DI. Inaccuracy in pediatric outpatient blood pressure measurement.Pediatrics 2007; I I9(3):e538-e543.

22. Menard SW, Park MK, Scholfield J.The San Antonio biethnic children's blood pressure study: anthropometric findings. Clin Exc Nur Pract 1999; 3:19-27.

23. Fixler DE, Kautz JA, Dana K. Systolic blood pressure differences among epidemiological studies. Hypertension 1980; 2(Suppl I):I3-I7.

24. Rocchini AP, Katch V,Anderson J. Blood pressure in obese adolescents: effects of weight loss. Pediatrics 1988; 82:16-23.

25. Baker JL, Olsen LW, Sorensen TI. Childhood body-mass index and the risk of coronary heart disease in adulthood. N Engl J Med 2007;357:23292337.

26. Mitsnefes M. Hypertension in children and adolescents. Pediatr Clin North Am 2006; 53:493-512. 